\title{
Ein 15-jähriger Fußballer mit Urtikaria, Fieber, Arthritis und Myositis
}

Es wird der Fall eines Jugendlichen berichtet, der anfänglich diagnostische Probleme mit sich brachte und dann unter Therapie zunächst eine deutliche Krankheitsverschlechterung zeigte, bevor es zu einer Besserung kam.

\section{Kasuistik}

Ein 15 Jahre alter Jugendlicher entwickelte seit Oktober 2017 eine Urtikaria und seit dem Silvesterabend 2017 abendliches Fieber und Appetitlosigkeit. Hinzu kamen innerhalb von 2 Monaten eine Gewichtsabnahme von $6 \mathrm{~kg}$, Gliederschmerzen und massive Einschlafprobleme. Es bestanden Schwellungen der PIP- und der DIP-Gelenke der 2. und 5. Finger beidseits mit jeweiliger Besserung innerhalb eines Tages. Dann entwickelte sich eine Knieschwellung rechts für 10 Tage. Bei der Blutuntersuchung in der Jugendarztpraxis zeigten sich eine Leukozytose von $24900 / \mu l$ mit Neutrophilie, die BSG mit $80 \mathrm{~mm} / \mathrm{h}$ deutlich beschleunigt und das CRP mit $190 \mathrm{mg} / \mathrm{l}$ sowie die LDH mit $675 \mathrm{U} / \mathrm{I}$ stark erhöht. Daraufhin erfolgte die Einweisung in unsere Klinik.

In der weiteren Vorgeschichte war es bereits im Winter 2016/2017 zu einer Urtikaria mit subfebrilen Temperaturen und kurzfristigen Gelenkschwellungen mit einer Besserung innerhalb von 2 Wochen gekommen. Der Jugendliche wurde in Südafrika als Kind einer HIV-positiven Mutter geboren und als Säugling nach Deutschland adoptiert. Bei einem Aufenthalt in Südafrika 2016 hatte er sich nicht in einem Malariaendemiegebiet aufgehalten. Der Jugendliche wies keine wesentlichen Vorerkrankungen auf und war aktiver Fußballspieler.

Bei Aufnahme in unsere Klinik im Januar 2018 sahen wir einen Jugendlichen in kaum reduziertem Allgemeinzustand, der auf beiden Beinen hüpfen konnte, mit leichter Urtikaria am linken Unterarm und der Hand ( $\triangleright$ Abb. 1). Im Verlauf traten auch juckende Hauterscheinungen an den Beinen auf ( $\triangleright$ Abb. 2). Der Knieumfang war rechts um $1 \mathrm{~cm}$ vermehrt und die Beugung mit $135^{\circ}$ gegen links $145^{\circ}$ leicht eingeschränkt bei freier Streckung. Die Finger und alle weiteren Gelenke waren frei beweglich,

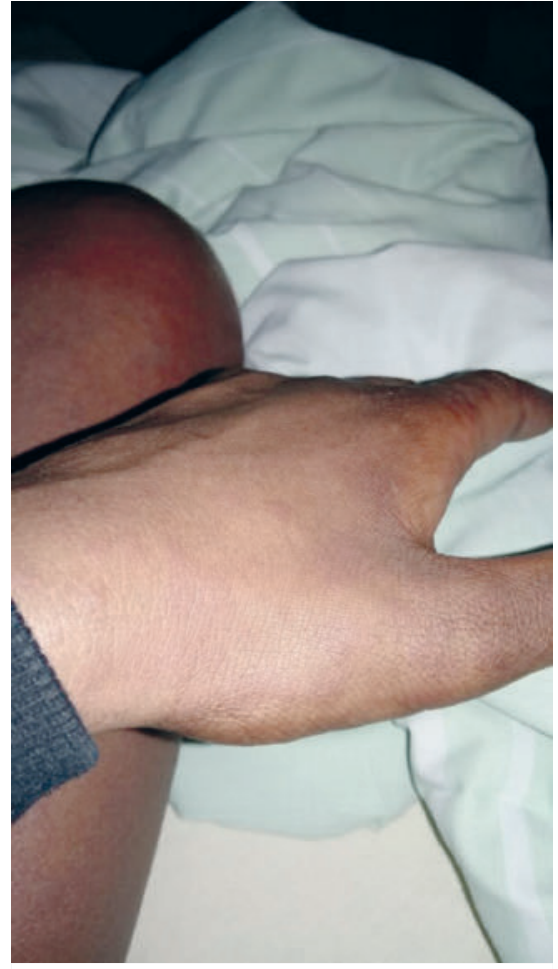

- Abb. 1 Exanthem bei Erstvorstellung in unserer Klinik an der Hand.

und der übrige klinische Untersuchungsbefund unauffällig.

Ein Röntgenbild des rechten Knies zeigte einen knöchern unauffälligen Befund, also auch keine Periostabhebung, bei sichtbarem Erguss, sonografisch fand sich neben einem $12 \mathrm{~mm}$ tiefen Erguss eine deutliche Synoviaverdickung. Bei uns war das CRP nur noch bei $127 \mathrm{mg} / \mathrm{l}$, aber deutlich erhöht, Procalcitonin normal bei $0,3 \mu \mathrm{g} / \mathrm{l}$, initiale Thrombozytose bis $568000 / \mu \mathrm{l}$, GOT mit 100, GPT mit 73, CK mit 697 und LDH mit $603 \mathrm{U} / \mathrm{l}$ alle erhöht. Auch das Troponin war mit $617 \mathrm{ng} / \mathrm{I}$ deutlich erhöht, ANA lagen bei 1:1280, DNS- und ENA-Ak waren negativ, IgG mit 19,5 g/l erhöht. Eine HIV-Infektion des Jugendlichen wurde erneut ausgeschlossen.

Die Punktion des rechten Kniegelenks zur Frage einer septischen Arthritis zeigte in der Synovialflüssigkeit 19200 Leukozyten/ $\mu$ mit 93 \% Neutrophilen. Die intravenöse antibiotische Therapie brachte keine Besserung der Fieberschübe. Die Kulturen aus Blut und Synovialflüssigkeit blieben steril. Die unter dem

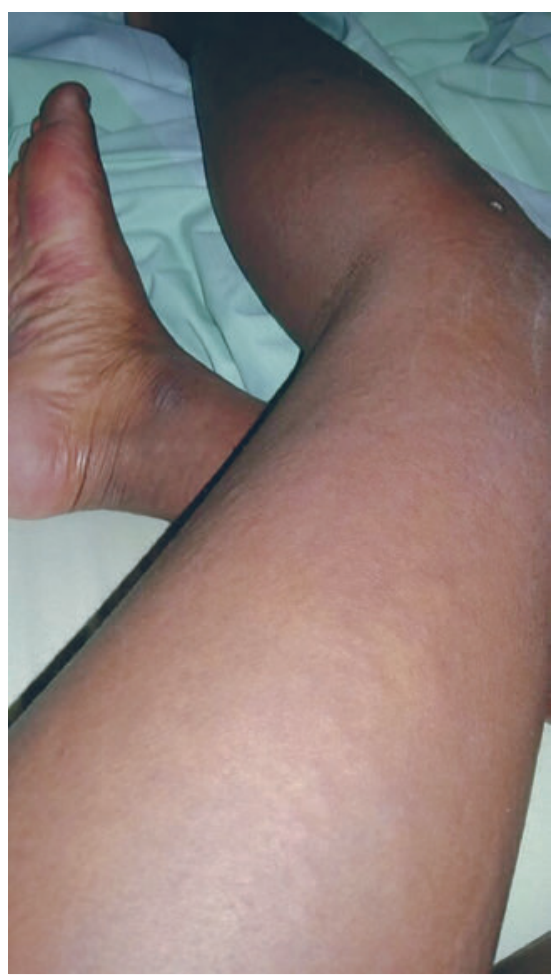

- Abb. 2 Exanthem an den Beinen in der 1. Woche des Klinikaufenthalts.

Verdacht auf eine Myositis mit Karditis im Verlauf mehrfach erfolgten Echokardiografien zeigten stets eine gute kardiale Funktion.

Nach Erhalt des stark erhöhten Serum-Calprotectins (= S100 A8/A9-Protein) von $78800 \mathrm{ng} / \mathrm{ml}$ begannen wir unter dem Verdacht auf eine systemische juvenile idiopathische Arthritis eine Therapie mit Anakinra $100 \mathrm{mg} /$ Tag. Hierunter kam es zum Anstieg des CRP auf $298 \mathrm{mg} / \mathrm{l}$, zur Verschlechterung des Allgemeinbefindens, Kreatininanstieg auf $251 \mu \mathrm{mol} / \mathrm{I}$ sowie Kreislaufinstabilität und Notwendigkeit der Verlegung auf die Intensivstation. Hier stieg die GOT auf maximal 2690, GPT 675, LDH 5325 U/l, es kam zur Ferritinerhöhung auf $65044 \mu \mathrm{g} / \mathrm{l}$, Leukozytopenie von minimal 1800/ $\mu$, Thrombozytopenie von minimal $105000 / \mu \mathrm{l}$, Fibrinogen bei $339 \mathrm{mg} / \mathrm{dl}$, Triglyzeride erhöht auf $275 \mathrm{mg} / \mathrm{dl}$ bei normaler Lebersyntheseleistung. Die Knochenmarkpunktion und eine Positronenemissionstomografie blieben ohne wegweisende Befunde. Unter der Diagnose eines Makrophagenaktivierungssyndroms erfolgte eine MethylprednisolonPulstherapie mit Fortsetzung der täglichen 
Anakinra-Gaben und anschließender oraler Prednisolon-Therapie mit $0,5 \mathrm{mg} / \mathrm{kg}$. Hierunter kam es zur Entfieberung, langsamen Besserung des Allgemeinzustands und weitgehender Besserung der Laborwerte bis zur Entlassung nach gut 5 Wochen.

Im weiteren Verlauf wurde die Therapie von täglichem Anakinra auf 4-wöchentliches Canakinumab umgestellt und die Prednisolon-Behandlung bis Mai 2018 ausschleichend beendet. Molekulargenetisch zeigte sich eine Heterozygotie für Varianten unklarer Signifikanz c.584 C> T; Thr195Met im NLRP3-Gen und c.279919 G > A im NOD2-Gen; die Untersuchung der übrigen bekannten Autoinflammationsgene IL1RN, IL36RN, LPIN2, MEFV, MVK, NLRC4, NLRP12, NLRP3, PLCG2, PSMB8, PSTIP1 und TNFRSF1A blieb unauffällig. Es kam zur Normalisierung der Muskel- bzw. Leberenzyme bis Dezember 2018 und zur Normalisierung des Serum-Calprotectins sowie zum langsamen Abfall und dann erst im Januar 2020 Verschwinden der ANA. Der Jugendliche spielt wieder im Verein Fußball. Das Canakinumab konnte im Juli 2019 abgesetzt werden, ohne dass es zu erneutem Fieber, Arthritis, Myositis oder Serumcalprotectin-Erhöhung im folgenden halben Jahr kam.

\section{Hintergrund}

Eine Myositis mit deutlichen Muskelenzymerhöhungen wie bei diesem Patienten im Rahmen einer systemischen juvenilen idiopathischen Arthritis ist sehr selten [1]. Das Auftreten eines Makrophagenaktivierungssyndroms auch nach Beginn der Therapie ist bei systemischer juveniler idiopathischer Arthritis auch unter Interleukin-1- oder Interleukin-6-Blockade beschrieben [2, 3].

\section{ZUSAMMENFASSUNG}

Berichtet wird von einem 15-Jährigen mit winterlicher Urtikaria, Fieberschüben, Arthritiden und Myositis, bei dem differenzialdiagnostisch an eine septische Arthritis, eine Kälteurtikaria und eine Autoimmunmyositis gedacht werden musste. Unter der Therapie einer systemischen juvenilen idiopathischen Arthritis mit Anakinra trat eine initiale dramatische Verschlechterung mit beginnendem akutem Nierenversagen und Kreislaufschwäche bei Makrophagenaktivierungssyndrom auf [4]. Es kam unter Methylprednisolon-Pulsen unter fortgesetzter Interleukin-1Blockade zur klinischen und paraklinischen Besserung. 18 Monate nach Beginn der Therapie konnte diese wieder beendet werden ohne bisherige Verschlechterung [5]. Der Patient wird regelmäßig nachbeobachtet.

\section{Interessenkonflikt}

Der Autor gibt an, Vortragshonorare der Firmen Novartis, Pfizer und AbbVie erhalten zu haben.

\section{Einhaltung ethischer Richtlinien}

Der Fall wurde als Poster bei der Jahrestagung der Gesellschaft für Kinder- und Jugendrheumatologie gemeinsam mit der Jahrestagung der Deutschen Gesellschaft für Rheumatologie im September 2019 in Dresden präsentiert.

Die Eltern und der Jugendliche haben der Veröffentlichung der Fotos zu medizinischen Fortbildungszwecken zugestimmt.

\section{Autor}

\section{Frank Dressler}

Kinderklinik der Medizinischen Hochschule Hannover, Klinik für Pädiatrische Pneumologie, Allergologie und Neonatologie, Kinder- und Jugendrheumatologische Ambulanz

\section{Korrespondenzadresse}

Dr. Frank Dressler

Kinderklinik der Medizinischen Hochschule Hannover

Klinik für Pädiatrische Pneumologie, Allergologie und Neonatologie, Kinder- und Jugendrheumatologische Ambulanz Carl-Neuberg-Str. 1, 30625 Hannover Dressler.frank@mh-hannover.de

\section{Literatur}

[1] De Benedetti F, Schneider R. Systemic juvenile idiopathic arthritis. In: Petty RE, Laxer RM, Lindsley CB, Wedderburn L, eds. Textbook of Pediatric Rheumatology. Philadelphia: Elsevier 2015; 205-216

[2] Ravelli A, Grom AA, Behrens EM, Cron RG. Macrophage activation syndrome as part of systemic juvenile idiopathic arthritis: diagnosis, genetics, pathophysiology, and treatment. Genes Immun 2012; 13 : 289-298

[3] Grom AA, Horne A, De Benedetti F. Macrophage activation syndrome in the era of biologic therapy. Nat Rev Rheumatol 2016; 12: 259-269

[4] Ravelli A, Minoia F, Davi S et al. 2016 Classification criteria for macrophage activation syndrome complicating systemic juvenile idiopathic arthritis: a European League against Rheumatism/American College of Rheumatology/Pediatric Rheumatology International Trials Organisation collaborative initiative. Ann Rheum Dis 2016; 75 : 481-489

[5] Maritsi DN, Vougioka O, Eleftheriou. Discontinuation of canakinumab following clinical disease remission is feasible in patients with systemic juvenile idiopathic arthritis. J Rheumatol 2020 Feb 15; doi:10.3899/jrheum.190011

Bibliografie

DOI https://doi.org/10.1055/a-1120-2235 arthritis + rheuma 2020; 40: 199-200 (c) Georg Thieme Verlag KG Stuttgart · New York ISSN 0176-5167 